\title{
Erratum
}

\section{A Dialkyldithiophosphate Derivative as Mild Steel Corrosion Inhibitor in Sulfuric Acid Solution [Int. J. Electrochem. Sci., 11 (2016) 4828 - 4839]}

Xiulan Su ${ }^{1}$, Chuan Lai ${ }^{1,2, *}$, Lincai Peng ${ }^{1}$,Hui Zhu ${ }^{1}$, Lvshan Zhou ${ }^{1}$, Lei Zhang, Xingqin Liu, Wei Zhang ${ }^{1}$

${ }^{1}$ School of Chemistry and Chemical Engineering, Sichuan University of Arts and Science, Dazhou 635000, PR China

${ }^{2}$ Material Corrosion and Protection Key Laboratory of Sichuan Province, Sichuan University of

Science and Engineering, Zigong 643000, PR China

*E-mail: laichuanemail@163.com

Received: 25 May 2016 / accepted: 25 May 2016 / Published: 4 June 2016

This is an erratum regarding the published article (Int. J. Electrochem. Sci., 11 (2016) 4828 - 4839). The authors would like to apologize for any inconvenience caused.

1. The affiliation of Lei Zhang and Xingqin Liu was not assigned in the paper and the correct affiliation should be "School of Chemistry and Chemical Engineering, Sichuan University of Arts and Science, Dazhou 635000, PR China.”.

2. The correct "ACKNOWLEDGEMENT" is as follows:

A Project Supported by Scientific Reserch Fund of SiChuan Provincial Education Department(No. 16ZA0358), the Opening Project of Material Corrosion and Protection Key Laboratory of Sichuan Province (No. 2015CL09), the Projects of Sichuan University of Arts and Science (Nos. HGXZ2015002, 2015TP002Z, X2015Z018), and the Opening Project of Key Laboratory of Green Catalysis of Sichuan Institutes of Higher Education (No. LYJ1503).

(C) 2016 The Author. Published by ESG (www.electrochemsci.org). This article is an open access article distributed under the terms and conditions of the Creative Commons Attribution license (http://creativecommons.org/licenses/by/4.0/). 